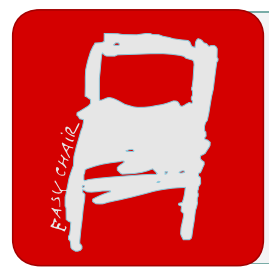

EPiC Series in Health Sciences

Volume 3, 2019, Pages 202-205

CAOS 2019. The 19th Annual Meeting of the International Society for Computer Assisted Orthopaedic Surgery

\title{
Navigation improves the ten to fifteen-year survival rate after total knee arthroplasty for severe coronal deformation. A French multicentric nationwide study
}

\author{
Jean-Yves Jenny, Dominique Saragaglia \\ University Hospital Strasbourg, Strasbourg, France \\ jean-yves.jennyechru-strasbourg.fr
}

\begin{abstract}
The survival rate of navigation-assisted total knee arthroplasty was superior to conventionally implanted after 12 years for knees with initial severe coronal deformation when mechanical revision was considered as the end-point. Longer follow-up is required to prove superiority of any technique.
\end{abstract}




\section{Introduction}

Total knee arthroplasty (TKA) is considered a highly successful procedure. Survival rates of more than $90 \%$ after 10 years are generally reported after implantation of conventional, fixed bearing TKAs. Restoring the limb alignment with an optimal ligament balance is considered critical (Insall 1983). Consequently, the long-term survival of conventional TKA by severe coronal deformation may be compromised. Various methods have therefore been described to treat major varus deformities (Laskin 1996), but there has so far been no consensus on the type of prosthesis to use, or the preferred ligament balancing technique. Some surgeons suggest using constrained and hinged prostheses, but these implants are associated with an increased complication rate. Computer navigation for TKA may help improving prosthesis alignment (Jenny 2005) especially in cases with severe coronal deformation (Saragaglia 2017).

The primary hypothesis of this study was that the 10-year survival rate of navigated TKAs for severe coronal deformation will be improved in comparison to conventional TKAs when analyzing survival rates and knee function as evaluated by the Knee Society Score (KSS).

\section{Material and methods}

All patients operated on between 2001 and 2004 in all participating centers for implantation of a TKA (whatever design used) were eligible for this study. Usual demographic and peri-operative items have been recorded. All patients were contacted after the 10-year follow-up for repeat clinical and radiological examination (KSS, Oxford knee questionnaire and knee plain X-rays). Patients who did not return were interviewed by phone call. For patients lost of follow-up, family or general practitioner was contacted to obtain relevant information about prosthesis survival. Conventional and navigated TKAs were paired according to age, gender, body mass index and severity of the coronal deformation (with steps of $5^{\circ}$ ). Survival curve was plotted according to the actuarial technique, using the revision for mechanical reason as end-point. The influence of the implantation technique was assessed with a logrank test at a 0.05 level of significance.

\section{Results}

1,604 TKAs were implanted during the study time-frame. 658 cases could be paired in conventional (329 cases) and navigated (329 cases) groups: in each group, 277 cases with a coronal deformation less than $10^{\circ}$ and 52 cases with a coronal deformation over $10^{\circ}$. There was no difference in any baseline criteria between conventional and navigated TKAs. 118 patients deceased before the 10 year follow up (18\%). Final follow-up was obtained for 382 cases (58\%). 15 prosthetic revisions were performed for mechanical reasons during the follow-up time (2\%) (table 1). The global survival rate after 13 years was $97 \%$. There was no significant difference between the 12-year survival rates of conventional (97\%) and navigated (98\%) TKAs in cases without severe coronal deformation. There was a significant difference between the survival rates of conventional (93\%) and navigated (98\%) TKAs in cases with severe coronal deformation. 
Table 1: Revised TKAs

\begin{tabular}{|l|c|c|c|c|}
\cline { 2 - 5 } \multicolumn{1}{c|}{} & \multicolumn{2}{c|}{ Conventional TKA } & \multicolumn{2}{c|}{ Navigated TKA } \\
\cline { 2 - 5 } \multicolumn{1}{c|}{} & Revised & Non revised & Revised & Non revised \\
\hline $\begin{array}{l}\text { No severe } \\
\text { coronal } \\
\text { deformation }\end{array}$ & 7 & 270 & 4 & 273 \\
\hline $\begin{array}{l}\text { Severe coronal } \\
\text { deformation }\end{array}$ & 3 & 49 & 1 & 51 \\
\hline
\end{tabular}

\section{Discussion}

This study confirms our initial hypothesis: navigated TKAs experienced a small but significant better long-term survival after 12 years in comparison to conventional TKAs when a severe preoperative coronal deformation was present, while no difference was observed in the opposite situation. A more consistent anatomical reconstruction and ligamentous balance of the knee with severe coronal deformation might be the explanation for this more consistent survival of the TKA in these challenging cases (Saragaglia 2017), while an optimal reconstruction is more consistently obtained with conventional implantation technique for less severe deformation. Navigated TKA may reproduce more consistently the normal knee kinematics, especially in these challenging cases (McClelland 2017).

This study suggests that navigation implantation should be the default technique for preoperative coronal deformation greater than $10^{\circ}$.

\section{References}

1. Insall JN, Hood RW, Flawn LB, Sullivan DJ., The total condylar knee prosthesis in gonarthrosis. A five to nine-year follow-up of the first one hundred consecutive replacements, J Bone Joint Surg Am 65, pp: 619-628, 1983.

2. Laskin RS, The Insall award. Total knee replacement with posterior cruciate ligament retention in patients with a fixed varus deformity. Clin Orthop 331, pp: 29-34, 1996.

3. Jenny JY, Clemens U, Kohler S, Kiefer H, Konermann W, Miehlke RK, Consistency of implantation of a total knee arthroplasty with a non-image-based navigation system: a case-control study of 235 cases compared with 235 conventionally implanted prostheses, J Arthroplasty, 20(7), pp: 832-839, 2005.

4. Saragaglia D, Sigwalt L, Gaillot J, Morin V, Rubens-Duval B, Pailhé R, Results with eight and a half years average follow-up on two hundred and eight e-Motion FP® knee prostheses, fitted using computer navigation for knee osteoarthritis in patients with over ten degrees genu varum, Int Orthop, 41(2), pp: 293-299, 2017. 
5. McClelland JA, Webster KE, Ramteke AA, Feller JA. Total knee arthroplasty with computerassisted navigation more closely replicates normal knee biomechanics than conventional surgery. Knee, 24(3), pp:651-656, 2017.

\section{Disclosure}

Jean-Yves JENNY receives royalties from AESCULAP, is a paid consultant for Exactech, is an unpaid consultant for FH Orthopedics, is member of the board of the CAOS-International Society, of the International Society for Technology in Arthroplasty and of the French Society for Hip and Knee Surgery. Dominique SARAGAGLIA receives royalties from AESCULAP 